\title{
Heterogeneity and Endogenous Nonlinearity in an Artificial Stock Model
}

\author{
Hongquan $\mathrm{Li}^{1,2}, *$, Wei Shang ${ }^{1}$, and Shouyang Wang ${ }^{1}$ \\ ${ }^{1}$ Laboratory of Management, Decision and Information Systems, Academy of Mathematics \\ and Systems Science, Chinese Academy of Sciences, Beijing, 100080, China \\ ${ }^{2}$ Department of Applied Mathematics and Physics, Graduate School of Informatics, \\ Kyoto University, Kyoto, 606-8501, Japan \\ \{Lhquan, shangwei , sywang\}@amss.ac.cn \\ Tel.: +86-10-62651381; +81-75-753-3568; Fax: +81-75-753-4756
}

\begin{abstract}
We present a nonlinear structural stock market model which is a nonlinear deterministic process buffeted by dynamic noise. The market is composed of two typical trader types, the rational fundamentalists believing that the price of an asset is determined solely by its fundamental value and the boundedly rational noise traders governed by greed and fear. The interaction among heterogeneous investors determines the dynamics and the statistical properties of the system. We find the model is able to generate time series that exhibit dynamical and statistical properties closely resembling those of the S\&P500 index, such as volatility clustering, fat tails (leptokurtosis), autocorrelation in square and absolute return, larger amplitude, crashes and bubbles. We also investigate the nonlinear dependence structure in our data. The results indicate that the GARCH-type model cannot completely account for all nonlinearity in our simulated market, which is thus consistent with the results from real markets. It seems that the nonlinear structural model is more powerful to give a satisfied explanation to market behavior than the traditional stochastic approach.
\end{abstract}

Keywords: Computational finance; Nonlinearity; Heterogeneous agents; Endogenous fluctuations.

\section{Introduction}

Modern finance is based on the standard paradigm of efficient market and rational expectations. The efficient market hypothesis postulates that the current price contains all available information and past prices cannot help in predicting future prices. Sources of risk and market fluctuations are exogenous. Therefore, in the absence of external shocks, prices would converge to a steady-state path which is completely determined by fundamentals and there are no opportunities for consistent speculative profits. In real markets, however, traders have different information on traded assets and process information differently, therefore the assumption of homogeneous ra-

* Corresponding author. 
tional traders may not be appropriate. The efficient market hypothesis motivates the use of random walk increments in financial time series modeling: if news about fundamentals is normally distributed, the returns on an asset will be normal as well. However, the random walk assumption does not allow the replication of some stylized facts of real financial markets, such as volatility clustering, fat tails (leptokurtosis), autocorrelation in square and absolute return, larger amplitude, crashes and bubbles.

Recently, finance literature has been searching for structural models that can explain such observed patterns in financial data. A number of models were developed which build on boundedly rational, non-identical agents [1-4]. Financial markets are considered as systems of interacting agents which continually adapt to new information. Heterogeneity in expectations can lead to market instability and complicated dynamics. As a result, prices and returns in such markets may deviate significantly from fundamentals.

In these heterogeneous agent models, different groups of traders coexist, having different beliefs or expectations about future prices of risky assets. Two typical trader types can be distinguished. The first type is the rational fundamentalists, believing that the price of an asset is determined solely by its fundamental value. The second typical trader type is the noise traders, chartists, or even technical analysts, believing that asset prices are not completely determined by fundamentals but that they may be predicted by simple technical trading rules. The literature on behavioral finance (for surveys see [5]) emphasizes the role of quasi-rational, overreacting, and other psychology factors including investor's emotions.

This paper builds on the model of [6], which is a deterministic behavioral stock market model with agents influenced by their emotions. In [6], the trading activity of the agents is characterized by greed and fear. They optimistically believe in booming markets, but panic if prices change too abruptly. Although the model is deterministic, it replicates several aspects of actual stock market fluctuations quite well. This paper is to extend this model in two ways. We introduce fundamentalists into the model to analyze how the interaction of different types of investors determines the dynamics and the statistical properties of the system. Further, an exogenous noise is added to the law of motion in order to mimic real market because noise and uncertainty play an important role in real financial markets.

This paper is divided into five sections. Section 2 presents our model with heterogeneous traders. The third section brings forward the simulation results and discusses the behavior of nonlinear dynamics. The final section provides a brief summary and some conclusions.

\section{The Model}

Let us consider a security with price $P_{t-1}$ (closed price) on the last trading period. Assume that this security is in fixed supply, so that the price is only driven by excess demand. Let us assume that the excess demand $D_{t}$ is a function of the last price $P_{t-1}$ and the present fundamental value $P_{f, t}$. A market maker takes a long position whenever the excess demand is negative and a short position whenever the excess demand 
is positive so as to clear the market. The market maker adjusts the price in the direction of the excess demand with speed equal to $\lambda^{M}$. Accordingly, the log of the price at the end of period $t$ is given as

$$
p_{t}=p_{t-1}+\lambda^{M} D_{t}\left(p_{t-1}, p_{f, t}\right)
$$

where $p_{f}$ denotes the $\log$ of the fundamental value. In order to introduce an exogenous news arrival process as a benchmark for the analysis of the resulting price dynamics, we make the assumption that $p_{f}$ follows a Wiener process, and hence,

$$
p_{f, t}=p_{f, t-1}+\varepsilon_{t}
$$

with $\varepsilon_{t} \sim N\left(0, \sigma_{\varepsilon}^{2}\right)$.

This specification makes sure that neither fat tails, volatility clustering, nor any kind of nonlinear dependence are brought about by the exogenous news arrival process. Hence, emergence of these characteristics in market prices would not be driven by similar characteristics of the news but would rather have to be attributed to the trading process itself. Agents' interactions magnify and transform exogenous noise into fat-tailed returns with clustered volatility.

The market is composed of two typical trader types. One type is the rational fundamentalists, believing that the price of an asset is determined solely by its fundamental value, and the other type is the boundedly rational traders whose behavior is influenced by their greed and fear. Let us assume that a fraction $\alpha$ of investors follows a fundamentalist strategy and a fraction $(1-\alpha)$ for boundedly rational traders. Let $D_{t}^{F}$ and $D_{t}^{N}$ be, respectively, the demands of fundamentalists and boundedly rational traders. The excess demand for the security is thus given by

$$
D_{t}=\alpha D_{t}^{F}+(1-\alpha) D_{t}^{N} \quad 0 \leq \alpha \leq 1
$$

Fundamentalists react to difference between price and fundamental value. The demand of fundamentalists in period $t$ is

$$
D_{t}^{F}=\lambda_{t}^{F}\left(p_{f, t}-p_{t-1}\right) \quad \lambda_{t}^{F}>0
$$

where $\lambda_{t}^{F}$ is a parameter that measures the speed of reaction of fundamentalist traders; we will assume that $\lambda_{t}^{F}=\lambda^{F}$ throughout the paper. This demand function implies that the fundamentalists believe that the price tends to the fundamental value in the long run and reacts to the percentage mispricing of the asset in a symmetric way with respect to underpricing and overpricing.

Following [6], the actions of the boundedly rational traders in our model are governed by greed and fear. The agents greedily take long positions because they know that the stock market increases in the long run. However, they also know the fact that stock prices always fluctuate, and such behavior is risky. The larger the risk, the more the agents reduce their investments. If prices change too strongly, they even panic and go short. For simplicity, it is assume that greed and fear-based behavior only occurs 
for two activity levels. As long as the market evolves stably, the agents are rather calm. However, in turbulent times both greed and fear increase. The emotional regime switching process may be formalized as follows:

$$
D_{t}^{N}=\lambda_{t}^{N}-\frac{2}{\lambda_{t}^{N}}\left(p_{t-1}-p_{t-2}\right)^{2}
$$

where $\lambda_{t}^{N}$ is a parameter that measures the activity level of emotional reaction to market volatility. If $\frac{1}{5} \sum_{i=1}^{5}\left|p_{t-i}-p_{t-i-1}\right| \leq 0.045$ and $\left|p_{t-1}-p_{t-1}\right| \leq 0.05$, then $\lambda_{t}^{N}=0.05$; otherwise, $\lambda_{t}^{N}=0.10$. In other words, the agents are rather calm if the average volatility in the last 5 trading periods is below 4.5 percent and if the most recent absolute log price change is below 5 percent. Otherwise the activity level increases from 0.05 to 0.10 . Similar to the explanation in Ref. [20], the first term of (5) reflects the greedy autonomous buying behavior of the agents whereas the second term of (5) captures the fear of the agents. Note that in the turbulent regime, extreme log price changes can be as large as \pm 10 percent. In the calm regime, extreme returns are restricted to \pm 5 percent.

\section{Simulation Results and Statistical Properties}

In this section, we analyze the statistical properties of the simulated time series, which have been generated with 2000 observations in each stochastic simulation in order to allow the system to get sufficiently close to the asymptotic dynamics and to have time series as long as the daily time series of the S\&P 500 index between 6 October 1999 and 14 September 2007. Fig.1 reports the time series plot of the S\&P500 and the simulation series generated by our model with parameters $\lambda^{M}=1, \lambda^{F}=1, \alpha=0.5, \sigma_{\varepsilon}=0.03$, and initial value $p_{0}=p_{f, 0}=6.12,\left[p_{1}, p_{2}, p_{3}, p_{4}, p_{5}\right]=[6.07,6.08,6.14,6.18,6.20]$. Table 1 reports the statistics of the daily returns on the S\&P500 and the model-generated time series. The Ljung-Box Q statistics for up to 30 lags for returns $(\mathrm{Q}(30))$ and squared returns $(\mathrm{QS}(30))$ are also presented. The $\mathrm{Q}(30)$ statistic for testing the hypothesis that all autocorrelations up to lag 30 are jointly equal to zero in the stock markets is greater than the value of $\chi^{2}$ distribution with 30 degrees of freedom at the $5 \%$ level, suggesting that the null hypothesis of the independence of returns should be rejected. Thus, linear serial dependencies seem to play a significant role in the dynamics of stock returns. The next and the most important question for the study of the behavior of nonlinear dependencies in stock returns, is: do these returns also exhibit nonlinear serial dependencies? The easiest way to answer this question is by examining the autocorrelation behavior of squared daily returns. The values of QS(30) (see Table 1) provide strong evidence of nonlinear dependence, indicating that the conditional distributions of the daily returns are changing through time. This is a symptom of ARCH effects. The results from Fig. 1 and Table 1 indicate that the model displays statistical properties similar to those of the S\&P500 index and can replicate the stylized facts of real financial markets, such as volatility clustering, 


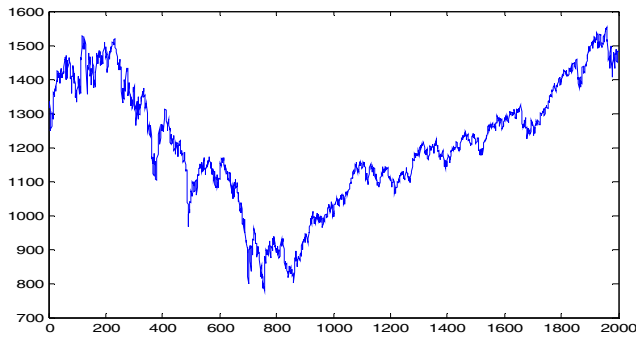

(a)

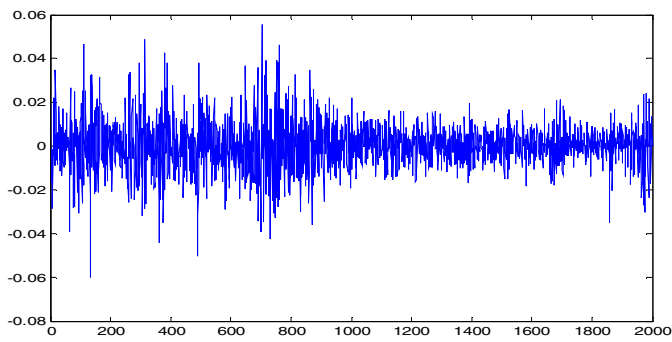

(b)

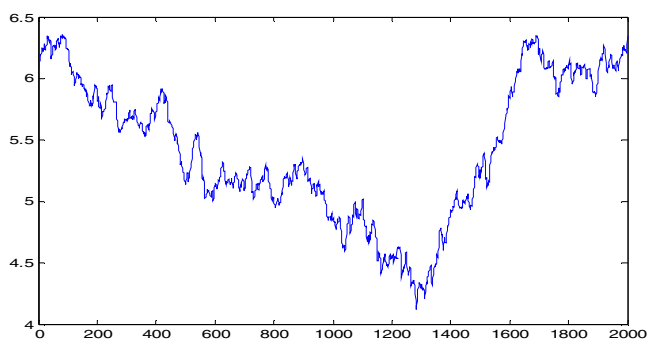

(c)

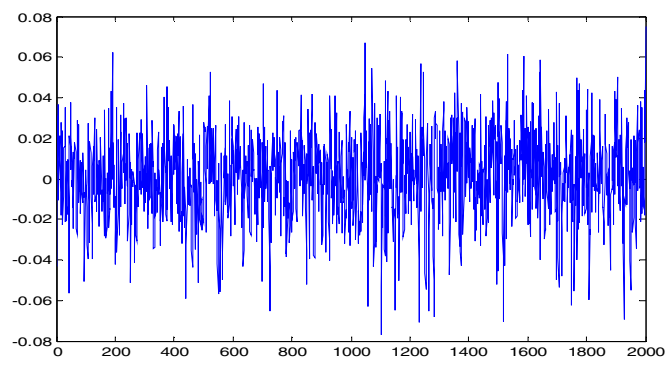

(d)

Fig. 1. Time series of the S\&P500 index (a), daily returns series of the S\&P500 (b), simulated price series (c) and simulated returns series (d) 
Table 1. Summary statistics of daily returns on the S\&P500 index and the simulated time series

\begin{tabular}{llllllll}
\hline Sample & Mean & Variance & Skewness & Kurtusis & Jar.Bra & Q(30) & QS(30) \\
\hline S\&P500 & 0.0001 & 0.0111 & 0.05 & 5.62 & 573 & 51 & 1434 \\
Model & 0.0001 & 0.0203 & -0.20 & 3.51 & 36 & 392 & 181 \\
\hline
\end{tabular}

excess kurtosis, autocorrelation in square return, crashes and bubbles. Furthermore, all these interesting features in our model arise endogenously from the trading process and interactions of our agents. With the assumptions of IID Normal innovations of the fundamental value, none of these characteristics can be attributed to exogenous influences.

The significant deviations from normality (larger Jar.Bra) and the significant QS(30) statistic in Table 1 suggest that there is very strong evidence of nonlinear structure in simulated return series. In order to detect the nonlinear dynamics deeply, we use the correlation dimension method and the BDS test.

The method of the correlation dimension introduced by Grassberger and Procaccia [7] provides an important diagnostic procedure for distinguishing between deterministic chaos and stochasticity in a time series. If the correlation integral $C(\varepsilon, m)$ measures the fraction of total number of pairs $\left(x_{i}, x_{i+1}, \cdots, x_{i+m-1}\right),\left(x_{j}, x_{j+1}, \cdots, x_{j+m-1}\right)$, such that the distance between them is no more than $\mathcal{E}$, then the correlation dimension is defined as:

$$
d_{c}=\lim _{\varepsilon \rightarrow 0} \frac{\ln C(\varepsilon, m)}{\ln \varepsilon}
$$

where

$$
C_{m, n}(\varepsilon)=\frac{1}{n(n-1)} \sum H\left(\varepsilon-\left\|X_{i}-X_{j}\right\|\right), i \neq j
$$

$H(u)$ is Heaviside step function, $H(u)=1$ if $u \geq 0,0$ otherwise; $n=$ the number of observations, $\mathcal{E}=$ distance, $C(\varepsilon, m)=$ correlation integral for dimension $m, X=$ the time series.

It is necessary to notice that when the embedding dimension $m$ increases, the dimension $d_{m}$ is reached, such that $d_{c}^{*}$ is the estimate of the true correlation:

$$
d_{c}^{*}=\lim _{m \rightarrow \infty} d_{c}(m)
$$

If $d_{m}$ tends to a constant as $m$ increases, then $d_{m}$ yields an estimate of the correlation dimension of the attractor, namely $d_{c}^{*}$.

In this case, the data are consistent with deterministic behaviour. If $d_{m}$ increases without bound as $m$ increases, this suggests that the data are either stochastic or noisy chaotic. 
The limitation of correlation dimension procedure is that no formal hypothesis testing is possible. To deal with the problems of using the correlation dimension test, Brock et al. [8] devised a new statistical test which is known as the BDS test. The BDS tests the null hypothesis of whiteness (independent and identically distributed observations) against an unspecified alternative using a nonparametric technique. The BDS test is a powerful test for distinguishing random system from deterministic chaos or from nonlinear stochastic systems. However, it does not distinguish between a nonlinear deterministic system and a nonlinear stochastic system. Essentially, the BDS test provides a direct (formal) statistical test for whiteness against general dependence, which includes nonwhite linear and nonwhite nonlinear dependence. Therefore, the null hypothesis of i.i.d. may be rejected because of non-stationarity, linear or nonlinear dependence or chaotic structure. The BDS statistic measures the statistical significance of the correlation dimension calculations. Brock et al. demonstrated that

$$
\left[C_{m, n}(\varepsilon)-C_{1, n}(\varepsilon)^{m}\right] \times \sqrt{n}
$$

is normally distributed with a mean of zero. The BDS statistic, $W$, that follows is normally distributed and is given by

$$
W=\sqrt{n}\left[C_{m, n}(\varepsilon)-C_{1, m}(\varepsilon)^{m}\right] / \sigma_{m, n}(\varepsilon)
$$

where $C_{m, n}(\varepsilon)$ and $C_{1, n}(\varepsilon)$ are given in (7) and $\sigma_{m, n}(\varepsilon)$ is an estimate of the standard deviation. $W$ converges in distribution to $N(0,1)$.

The BDS statistic can be used to test the residuals of GARCH type models for independence. If the null model is indeed GARCH then the standardized residuals of the fitted GARCH model should be independent. However, it was demonstrated by Hsieh [9] that the distribution of the $W$ statistic changes when applied on the residuals of ARCH and GARCH-type filtered. Therefore, we have to use the simulated distribution of the BDS statistic by bootstrap method (which can be easily handled with Eviews5.0) in such cases.

Before proceeding any further, we should firstly test the stationarity of our modelgenerated data. The augmented Dickey-Fuller unit-root test (ADF) shows that one unit root exists in the simulated price series $\left(p_{t}\right)$. The $\mathrm{ADF}$ value is -1.02 , which is greater than the critical value at $5 \%$. However, the $\mathrm{ADF}$ value for returns series (the difference of $p_{t}$ ) is -22.44 , which is less than the critical value at $5 \%$. The unit root test strongly rejects a unit root for simulated returns series and we can conclude that the returns series is stationary. Given stationarity in returns, none of non-IID behaviour of stock returns can be attributed to non-stationarity.

The correlation dimension estimates for the simulated returns series as well as the S\&P500 index returns series are presented in Table 2. Clearly, our model has correlation dimension estimates similar to that of the S\&P500. For the two series, the correlation dimension estimates are lower than the embedding dimensions; however, they do not converge to a stable value. The results indicate the underlying process is nether purely random or completely chaotic. 
Table 2. Estimates of the correlation dimension for the embedding dimension $m$

\begin{tabular}{llllllllll}
\hline$m$ & 2 & 3 & 4 & 5 & 6 & 7 & 8 & 9 & 10 \\
\hline S\&P500 & 1.80 & 2.63 & 3.26 & 3.98 & 4.35 & 5.03 & 5.44 & 5.68 & 6.09 \\
Model & 1.68 & 2.38 & 3.19 & 3.96 & 4.33 & 5.04 & 5.60 & 6.04 & 6.48 \\
\hline
\end{tabular}

We now apply the BDS test to the simulated returns series. In order to eliminate linear structure in our model, the returns data were firstly filtered by an Auto-regressive Moving Average method whose lag length was determined by the Akaike information criterion.

Table 3 gives the results of BDS tests. They strongly reject the hypothesis that simulated stock returns are IID. The rejection of IID is consistent with the view that stock returns are generated by nonlinear stochastic systems, e.g. ARCH and GARCH-type models, or nonlinear deterministic process such as chaos or noisy chaotic model. ARCH-type models have been widely used to describe conditional heteroskedasticity and are deemed to closely resemble the typical behavior of stock market volatility. Our interesting question is: Does the conditional heteroskedasticity captured by ARCH-type models account for all the nonlinearity in our model which is a nonlinear deterministic process disturbed by dynamic noise?

To answer this question, we ran the BDS procedures on the standardized residuals of the fitted GARCH model to test if GARCH captures all nonlinear dependence in stock returns.

The best GARCH model is determined by AIC criterion. The model is:

$$
\text { Mean equation } r_{t}=0.2279 r_{t-2}+0.3397 \varepsilon_{t-1}+\varepsilon_{t}
$$

$$
\text { Variance equation } \sigma_{t}^{2}=0.0001+0.2565 \varepsilon_{t-1}^{2}+0.4126 \sigma_{t-1}^{2}
$$

$$
\text { (6.58) (6.72) }
$$

$$
\mathrm{Q}(10)=4.61 \quad \mathrm{QS}(10)=13.55
$$

The parentheses contain the z-statistics of the estimated coefficients. The Q(10) and QS(10) statistics of the standardized residuals are significantly smaller than the critical value 18.31 at $5 \%$, indicating the absent of linear autocorrelation and heteroskedasticity. The standardized residuals from GARCH model serve as the filtered data.

Table 4 shows that the BDS statistics on the standardized residuals are much smaller than those of the ARMA filtered data. However, most BDS statistics are still outside the $5 \%$ critical range. There is sufficient evidence to indicate that the GARCH model cannot completely account for all nonlinearity in the simulated returns. This evidence is thus consistent with the results reported by Hsieh [9] in which Hsieh found that the popular GARCH-type models couldn't capture all nonlinear dependence in 10 stock returns including the weekly S\&P500 index and the daily S\&P500 index. This suggests that the nonlinear deterministic model disturbed by dynamic noise may be more powerful to mimic and explain the observed fluctuations in real economic and financial time series than the traditional nonlinear stochastic models.

Finally, it should be noted that we only display one simulation run here for different parameter values. Because of random element added to our model, one simulated 
Table 3. BDS statistics for ARMA filtered data ${ }^{a}$

\begin{tabular}{lllll}
\hline Epsilons/sigma & 0.5 & 1 & 1.5 & 2 \\
\hline $\mathrm{m}=2$ & 12.2 & 11.6 & 10.4 & 8.36 \\
$\mathrm{~m}=3$ & 16.9 & 15.4 & 13.8 & 11.5 \\
$\mathrm{~m}=4$ & 18.4 & 15.8 & 13.7 & 11.3 \\
$\mathrm{~m}=5$ & 18.5 & 15.7 & 13.5 & 11.2 \\
\hline
\end{tabular}

all significant at $5 \%$ (two-tailed) level.

Table 4. BDS statistics for GARCH standardized residuals

\begin{tabular}{lllll}
\hline Epsilons/sigma & 0.5 & 1 & 1.5 & 2 \\
\hline $\mathrm{m}=2$ & $3.70^{*}$ & $3.00^{*}$ & 1.83 & 0.21 \\
$\mathrm{~m}=3$ & $6.25^{*}$ & $4.99^{*}$ & $3.48^{*}$ & 1.53 \\
$\mathrm{~m}=4$ & $6.42^{*}$ & $4.63^{*}$ & $2.91^{*}$ & 0.66 \\
$\mathrm{~m}=5$ & $6.73^{*}$ & $4.32^{*}$ & $2.60^{*}$ & 0.51 \\
\hline
\end{tabular}

*significant at $5 \%$ (two-tailed) level.

data may differ from each other. However, the nonlinear dynamical behavior and statistical properties for the fixed parameter can be relatively stable. As revealed in further simulations, our conclusions are quite robust (which may easily be checked).

\section{Conclusion}

In this paper we have outlined a nonlinear deterministic model disturbed by dynamic noise. The dynamical system is able to generate some stylized facts present in real markets: excess kurtosis, volatility clustering, and autocorrelation in square and absolute return, crashes and bubbles. The market is composed of two typical trader types, the rational fundamentalists believing that the price of an asset is determined solely by its fundamental value and the boundedly rational traders governed by greed and fear. The interaction of different types of investors determines the dynamics and the statistical properties of the system.

It is worth emphasizing that, in our model, all these interesting qualitative features arise endogenously from the trading process and the interactions of heterogeneous agents. With the assumption of IID normal innovations of the fundamental value, none of these characteristics can be attributed to exogenous impacts. Taking together the complex behavior in real stock markets and the academic achievements (see [1-2], [10]), it seems more robust than the traditional stochastic approach to model the observed data by a nonlinear structural model buffeted by dynamic noise.

Acknowledgments. This research was supported by the MEXT Global COE Program on Informatics Education and Research Center for Knowledge-Circulating society (Kyoto University), the China Postdoctoral Science Foundation, the National Natural Science Foundation of China and Scientific Research Fund of Hunan Provincial Education Department. The authors would like to thank Prof. Masao Fukushima for his helpful comments and suggestions. All errors remain, of course, our own responsibility. 


\section{References}

1. Brock, W.A., Hommes, C.H.: Heterogeneous beliefs and routes to chaos in a simple asset pricing model. Journal of Economic Dynamics and Control 22, 1235-1274 (1998)

2. Hommes, C.H., Manzan, S.: Comments on Testing for nonlinear structure and chaos in economic time series. Journal of Macroeconomic 28, 169-174 (2006)

3. Gaunersdorfer, A.: Endogenous fluctuations in a simple asset pricing model with heterogeneous agents. Journal of Economic Dynamics and Control 24, 799-831 (2000)

4. Malliaris, A.G., Stein, J.L.: Methodological issues in asset pricing: random walk or chaotic dynamics. Journal of Banking and Finance 23, 1605-1635 (1999)

5. Shiller, R.J.: The irrationality of markets. The Journal of Psychology and Financial Markets 3, 87-93 (2002)

6. Westerhoff, F.: Greed, fear and stock market dynamics. Physica A 343, 635-642 (2004)

7. Grassberger, P., Procaccia, I.: Characterization of strange attractors. Physical Review Letters 50, 346-349 (1983)

8. Brock, W.A., Dechert, W.D., Scheinkman, J.A., LeBaron, B.: A test for independence based on the correlation dimension. Econometric Reviews 15, 197-235 (1996)

9. Hsieh, D.A.: Chaos and nonlinear dynamics: application to financial markets. Journal of Finance 46, 1839-1877 (1991)

10. Kyrtsou, C., Terraza, M.: Stochastic chaos or ARCH effects in stock series? A comparative study. International Review of Financial Analysis 11, 407-431 (2002) 